\title{
CONVEX QUADRANGULATIONS OF BICHROMATIC POINT
} SETS

\author{
Alexander Pilz* \\ Institute of Software Technology, Graz University of Technology, Graz, Austria. \\ apilz@ist.tugraz.at \\ Carlos Seara ${ }^{\dagger}$ \\ Department of Mathematics, Universitat Politècnica de Catalunya, Barcelona, Spain. \\ carlos.seara@upc.edu \\ Received (received date) \\ Revised (revised date) \\ Communicated by (name)
}

\section{ABSTRACT}

\begin{abstract}
We consider quadrangulations of red and blue points in the plane where each face is convex and no edge connects two points of the same color. In particular, we show that the following problem is NP-hard: Given a finite set $S$ of points with each point either red or blue, does there exist a convex quadrangulation of $S$ in such a way that the predefined colors give a valid vertex 2-coloring of the quadrangulation? We consider this as a step towards solving the corresponding long-standing open problem on monochromatic point sets.

Keywords: quadrangulation, bichromatic point set, NP-completeness
\end{abstract}

\section{Introduction}

A quadrangulation of a set $S$ of $n$ points in the Euclidean plane is a partition of 26 the convex hull of $S$ (denoted by $\mathrm{CH}(S)$ ) into quadrangles (i.e., 4-gons) such that 27 the union of the vertices of the quadrangles is exactly the point set $S$, and two 28 quadrangles share either a common vertex, a common edge, or no point at all. 29 Hence, the quadrangulation is also a geometric (straight-line) planar graph with

* Supported by a Schrödinger fellowship, Austrian Science Fund (FWF): J-3847-N35.

${ }^{\dagger}$ Supported by projects Gen. Cat. DGR 2014SGR46 and MINECO MTM2015-63791-R.

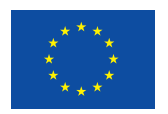

This project has received funding from the European Union's Horizon 2020 research and innovation programme under the Marie Skłodowska-Curie grant agreement No 734922 . 
vertex set $S$. A quadrangulation is a convex quadrangulation if every quadrangle is convex.

It is well-known that a point set admits a quadrangulation if and only if the number of points on the convex hull is even. In particular, given a set $S$ of size $n$ with $h$ points on its convex hull, i.e., $|\mathrm{CH}(S)|=h$, the number of edges in any triangulation of $S$ is $2 n-2-\frac{h}{2}$, and the corresponding number of faces is $n-1-\frac{h}{2}$. Not every such set admits a convex quadrangulation, and deciding this in polynomial time is an open problem (tracing back to Joe Mitchell in $1993^{16}$ ).

A graph is vertex $k$-colorable (in brief $k$-colorable) if there exists a mapping of each vertex of the graph to exactly one of $k$ colors such that no two vertices of the same color share an edge. A 2-colorable graph is a bipartite graph. It is known that every quadrangulation is bipartite.

A bichromatic point set is a finite set $S$ of points together with a mapping of each point to one of two colors. Throughout this paper, these colors will be red and blue.

Our main question is whether for a given bichromatic point set there is a convex quadrangulation such that the colors of the points define a valid 2-coloring of the quadrangulation. We call such a quadrangulation valid. Consider a 2-coloring of any quadrangulation. There are at least two vertices of each color, and it is easy to construct examples of quadrangulations with arbitrarily many vertices that have only two vertices of one color. In Section 2, we show that this bound differs for convex quadrangulations. In Section 3, using observations of Section 2, we prove that deciding whether a bichromatic point set has a valid convex quadrangulation is NP-complete. The motivating question whether a (monochromatic) point set admits a convex quadrangulation is left open.

Next, we survey some of the main known results about quadrangulations.

Quadrangulations Quadrangulations of point sets or polygons were discussed by many authors; see the survey by Toussaint ${ }^{16}$. Since not all polygons or point sets admit quadrangulations, even when the quadrangles are not required to be convex, the author surveys results characterizing those planar sets that always admit quadrangulations (convex and non-convex) for quadrangulations of orthogonal polygons, simple polygons, and point sets.

Lubiw $^{13}$ shows that determining whether a simple polygon with holes has a convex quadrangulation is NP-complete. In contrast to that, there is a polynomialtime algorithm for a generalized variant of rectilinear polygons.

Bose and Toussaint ${ }^{3}$ show that a set $S$ of $n$ points admits a quadrangulation if and only if $S$ has an even number of extreme points. They present an algorithm that computes a quadrangulation of $S$ in $O(n \log n)$ time even in the presence of collinear points, adding an extreme Steiner point if necessary. If $S$ does not admit a quadrangulation, then their algorithm can quadrangulate $S$ with the addition of one extra point. 
Ramaswami, Ramos, and Toussaint ${ }^{14}$ show that a triangulated simple $n$-gon $P$ can be quadrangulated in linear time with the least number of outer Steiner points required for that triangulation, and that $\left\lfloor\frac{n}{3}\right\rfloor$ outer Steiner points are sufficient, and sometimes necessary, to quadrangulate $P$. They further show that $\left\lfloor\frac{n}{4}\right\rfloor$ inner Steiner points (and at most one outer Steiner point) are sufficient to quadrangulate $P$, and this can be done in linear time. The method can be used to quadrangulate arbitrary triangulated domains.

Convex quadrangulations Most of the work on convex quadrangulations is concerned with Steiner points. For example, Bremner et al. ${ }^{4}$ prove that if the convex hull of $S$ has an even number of points, then by adding at most $\frac{3 n}{2}$ Steiner points in the interior of its convex hull, we can always obtain a point set that admits a convex quadrangulation. The authors also show that $\frac{n}{4}$ Steiner points are sometimes necessary. Heredia and Urrutia ${ }^{9}$ improve these upper and lower bounds to $\frac{4 n}{5}+2$ and $\frac{n}{3}$, respectively.

Deciding in polynomial time whether a given (monochromatic) point set admits a convex quadrangulation without adding Steiner points seems to be a long-standing open problem. Only fixed-parameter-tractable algorithms and heuristics are known. Fevens, Meijer, and Rappaport ${ }^{8}$ present a polynomial-time algorithm to determine whether a point set $S$ admits a convex quadrangulation if $S$ is constrained to lie on a constant number of nested convex polygons. Schiffer, Aurenhammer, and Demuth ${ }^{15}$ propose a simple heuristic for computing large subsets of convex quadrangulations on a given point set.

Quadrangulations of colored point sets Cortés et al. ${ }^{6}$ discuss aspects of quadrangulations of bichromatic point sets. They study bichromatic point sets that admit a quadrangulation, and whether, given two quadrangulations of the same bichromatic point set, it is possible to transform one into the other using certain local operations. They present a family of 2-colorings, called onion 2-coloration (which is a 2-coloration of a point set such that all its convex layers have an even number of points with alternate colors), that are quadrangulatable and for which the graph of quadrangulations is always connected. They show that any bichromatic point set with convex layers having an even number of points with alternate colors has a valid quadrangulation, and any two such quadrangulations can be transformed into each other.

Alvarez, Sakai, and Urrutia ${ }^{2}$ prove that a bichromatic set $S=R \cup B$, where $R$ is the set of red points, $B$ is the set of blue points, and $|\mathrm{R}|=|\mathrm{B}|=n$, can be quadrangulated by adding at most $\left\lfloor\frac{n-1}{3}\right\rfloor+\left\lfloor\frac{n}{2}\right\rfloor+1$ Steiner points and that $\frac{m}{3}$ Steiner points are occasionally necessary, where $m$ is the number of quadrilaterals of the quadrangulation. They also show that there are 3-colored point sets with an even number of extreme points that do not admit a quadrangulation, even after adding Steiner points in the interior of the convex hull. 
Kato, Mori, and Nakamoto ${ }^{10}$ define the winding number $\omega(S)$ for a 3-colored point set $S$, and prove that a 3 -colored set $S$ of $n$ points in general position with a finite set $P$ of Steiner points added is quadrangulatable if and only if $\omega(S)=0$. When $S \cup P$ is quadrangulatable, then $|P| \leq \frac{7 n+34 m-48}{18}$, where the number of extreme points is $2 m$. This line of research is continued by Alvarez and Nakamoto ${ }^{1}$, who study $k$-colored quadrangulation of $k$-colored sets of points, where $k \geq 2$. Since not every set of points admits a $k$-colored quadrangulation, the use of Steiner points (choosing the color among the $k$ colors) is required in order to obtain one. They show that if $\omega(S)=0$ or $k \geq 4$, then a $k$-colored quadrangulation of $S$ can always be constructed using less than $\frac{(16 k-2) n+7 k-2}{39 k-6}$ Steiner points. (The authors note that $\omega(S)=0$ for any bichromatic $S$ where red and blue points on $\mathrm{CH}(S)$ alternate.)

\section{The red and the blue graph of a convex quadrangulation}

Let $Q$ be a convex quadrangulation with a valid red-blue coloring of its $n$ vertices. For every quadrangle, one diagonal connects the two red vertices of the quadrangle, and the other connects the two blue ones. We call them the red diagonal and the blue diagonal, respectively. Let $G_{\mathrm{R}}$ be the graph whose vertices are the red vertices of $Q$ and whose edges are the red diagonals of all quadrangles of $Q$. Let $G_{\mathrm{B}}$ be defined analogously. Since the colors are interchangeable, all the following statements hold equally for both graphs.

Lemma 1. $G_{\mathrm{R}}$ is a simple plane connected graph.

Proof. $G_{\mathrm{R}}$ is simple and plane as every red edge has its own quadrangle and the faces (quadrangles) are convex. Suppose that $G_{\mathrm{R}}$ is not connected. Then there exists a Jordan curve splitting the convex hull of $S$ that separates the red points and does not intersect a red edge. Further, there exits such a curve that intersects every quadrangle of $Q$ in at most one connected component. Consider an edge $e$ of $\mathrm{CH}(S)$ intersected by the curve. The two endpoints of $e$ have different colors and $e$ is adjacent to a quadrangle $q$. Since the curve does not intersect a red edge, one blue point $b$ of $q$ is separated from the other points of $q$. Due to our assumption, there has to be a red point on the same side of the curve as $b$, and therefore there is another quadrangle $q^{\prime}$ sharing an edge with $q$ that is intersected by the curve. However, $q^{\prime}$ can again only have $b$ as the only point on one side of the curve. Continuing this process until the curve reaches again the boundary of the convex hull, we see that it only separates $b$ from the remaining points, a contradiction.

Lemma 2. Every minimal cycle of $G_{\mathrm{R}}$ contains exactly one blue point, and every inner blue point is contained in a minimal cycle of $G_{\mathrm{R}}$. Blue extreme points are separated from the remaining set by a path in $G_{\mathrm{R}}$.

Proof. Consider the quadrangles that are adjacent to an inner blue point. The red diagonals of the quadrangles form a cycle that contain the blue point. Further, 
consider any minimal cycle of $G_{\mathrm{R}}$ and any edge therein. This edge corresponds to a quadrangle and there is one blue point of the quadrangle on each side of the edge. Observe that every blue point on the convex hull boundary is separated by a red path from the other blue vertices.

Theorem 1. Let $n_{\mathrm{R}}$ and $n_{\mathrm{B}}$ be the number of red and blue vertices, respectively, of a 2-colored convex quadrangulation. Then $n_{\mathrm{B}} \leq 2 n_{\mathrm{R}}-2$.

Proof. Observe that $G_{\mathrm{R}}$ and $G_{\mathrm{B}}$ have the same number $e$ of edges. By Euler's Polyhedral Formula we have

$$
n_{\mathrm{B}}-e+f_{\mathrm{B}}=2,
$$

where $f_{\mathrm{B}}$ is the number of faces in the blue graph (including the outer face). Lemma 2 implies $n_{\mathrm{R}}=f_{\mathrm{B}}-1+\frac{h}{2}$, where $h$ is the number of extreme points. Hence, we get

$$
n_{\mathrm{B}}+n_{\mathrm{R}}-\frac{h}{2}-1=e
$$

Since $G_{\mathrm{R}}$ is a plane geometric graph, we have $e \leq 3 n_{\mathrm{R}}-3-\frac{h}{2}$. By plugging this into the previous equation we get the claimed inequality.

Note that the inequality $e \leq n_{\mathrm{R}}-3-\frac{h}{2}$ is tight if and only if $G_{\mathrm{R}}$ is a triangulation. Fig. 1 shows an example where the bound is tight.

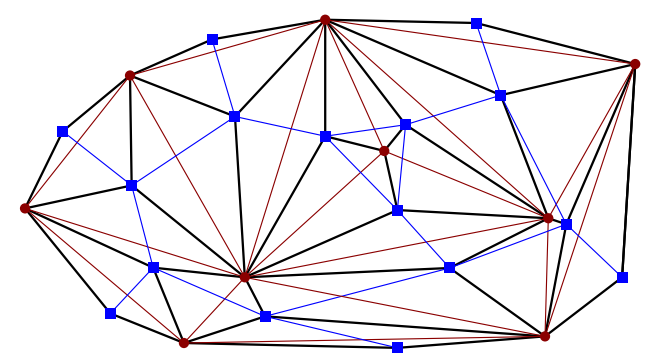

Fig. 1. An example showing that the bound on the relation between the red (round) and blue (squared) points in a convex quadrangulation (with thick black edges) is tight.

The structure of the red and the blue graph reveals a necessary condition of a bichromatic point set that allows a convex quadrangulation: Every segment between two red points must be intersected by a segment between two blue points. Cortés et al. ${ }^{5}$ give a quadratic-time algorithm to check for this property. However, this condition is not sufficient. 


\section{NP-completeness}

In this section we prove that the problem of deciding whether there exists a valid convex quadrangulation of a given bichromatic point set is NP-hard. Our reduction is from planar 3-SAT (cf. Ref. 12). The construction is based in large parts on placing two red points sufficiently close to a crossing between two segments between blue points, such that exactly one of these blue segments is a diagonal of a quadrilateral in any convex quadrangulation, and that the state of variables is propagated between the gadgets. We show that once there is a valid choice of these blue diagonals (corresponding to a satisfying variable assignment), they are part of a valid convex quadrangulation, and argue that the construction has coordinates of polynomial size.

In a planar 3-SAT instance, we are given a Boolean formula in conjunctive normal form; the corresponding incidence graph consists of variables and clauses as vertices, in which an edge between a variable and a clause indicates an occurrence, and which is known to be planar. As common in this type of reductions, we transform an embedding of the incidence graph of a planar 3-SAT instance to a bichromatic point set by replacing elements of the graph drawing by gadgets. For simplicity, we may consider the drawing to consist of edges that are represented by a sequence of orthogonal line segments (actually, one bend suffices, see Ref. 11). An edge in this drawing carries the truth value of a variable to the clause gadgets (possibly via a negation).

\subsection{Gadgets}

Each edge of the incidence graph are is represented by a chain of link gadgets. Each link gadget contains four blue points in convex position and two red points close to the crossing they define. Hence, one of the two blue edges must be a diagonal in any valid convex quadrangulation $Q$ (if it exists). See Fig. 2. If one of the segments is a diagonal of $Q$ (say, the one from bottom-left to top-right), the link gadget carries true (and the line segment is called the T-diagonal of the link gadget); if the other segment (being called the $F$-diagonal) is a diagonal of $Q$, the edge gadget carries false. Two of these links are joined such that the T-diagonal of the previous link crosses the F-diagonal of the next link and vice versa, and thus $Q$ cannot have a T-diagonal and an F-diagonal in the same edge gadget.

The gadgets for variables, bends, and negations are shown in Figures 3, 4, and 5, respectively, together with a possible valid convex quadrangulation and more detailed descriptions in the caption.

A variable gadget works by connecting three edge gadgets in a way that they all have either the T-diagonal or the F-diagonal as a diagonal; an arbitrary number of edges from the same variable vertex can be connected in that way. The variable gadget is shown in Fig. 3. Further, we need bends in the edge gadgets to connect horizontal and vertical parts, as well as negation gadgets. All of these are mere 

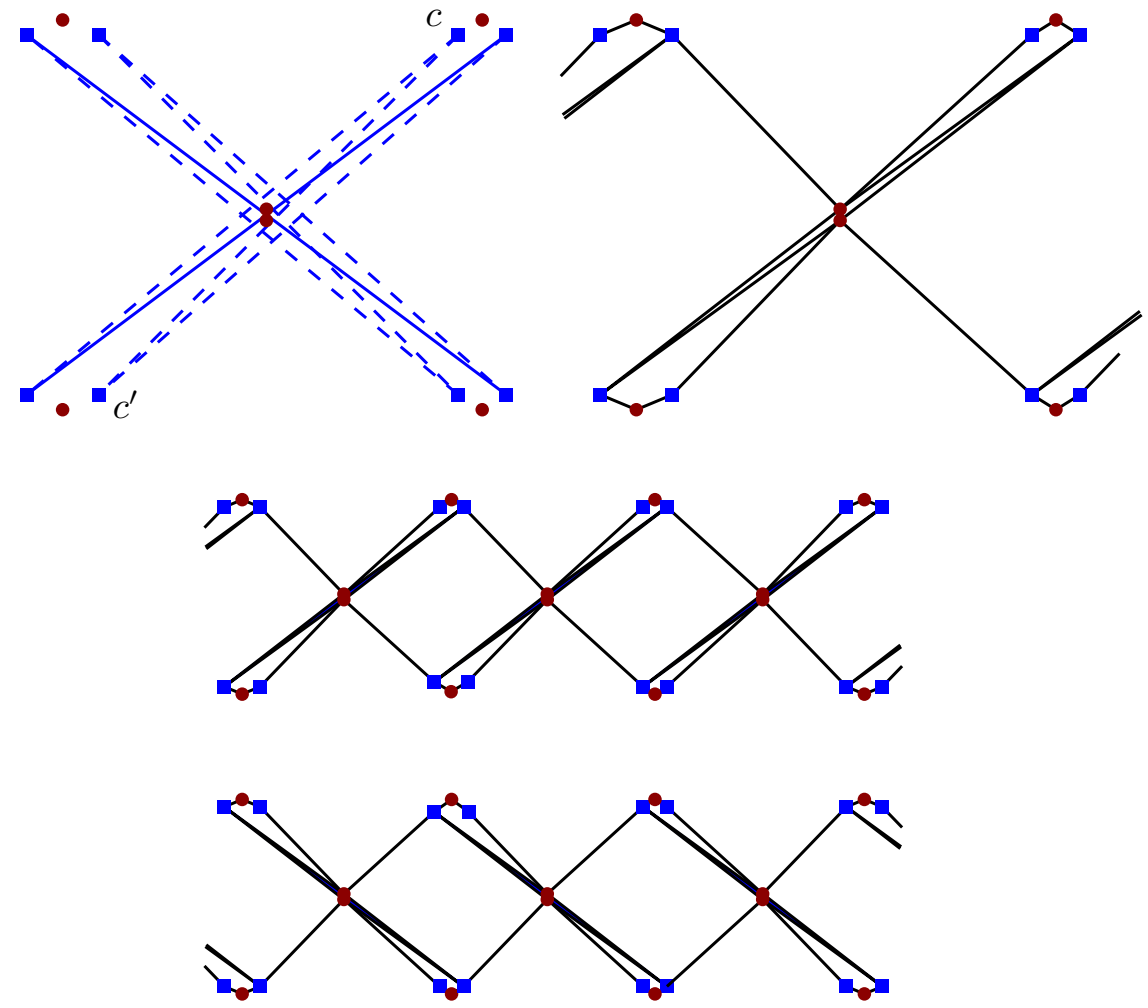

Fig. 2. A link gadget to model edges in the graph. The middle red points are that close to the crossing of the solid blue segments such that there is no other blue segment passing between them (as indicated by the dashed lines). (Note that to this end, the three-point "caps" at the ends of the segments have to have slightly different width, like those indicated by $c$ and $c^{\prime}$.) Exactly one of the blue segments has to be a diagonal of the quadrangulation, and combining these links propagates that decision. A possible quadrangulation is shown to the right. The link gadgets can be concatenated to form edges, as shown below.

appropriate combinations of link gadgets, figures and exact descriptions of these gadgets are provided in the appendix.

A clause gadget is shown in Fig. 6. In its center, there are two red points that are intersected by exactly three blue segments. There is exactly one combination of the diagonals of the three involved link gadgets that prevents each of these blue segments to become a diagonal, the one where all three link gadgets carry false. Fig. 7 shows valid convex quadrangulations for gadgets representing satisfied clauses for all seven possible variable settings.

\subsection{Quadrangulating the remaining parts of the convex hull}

For quadrangulating the parts of the convex hull between the gadgets, note that these regions are simple polygons with red and blue vertices alternating on the 

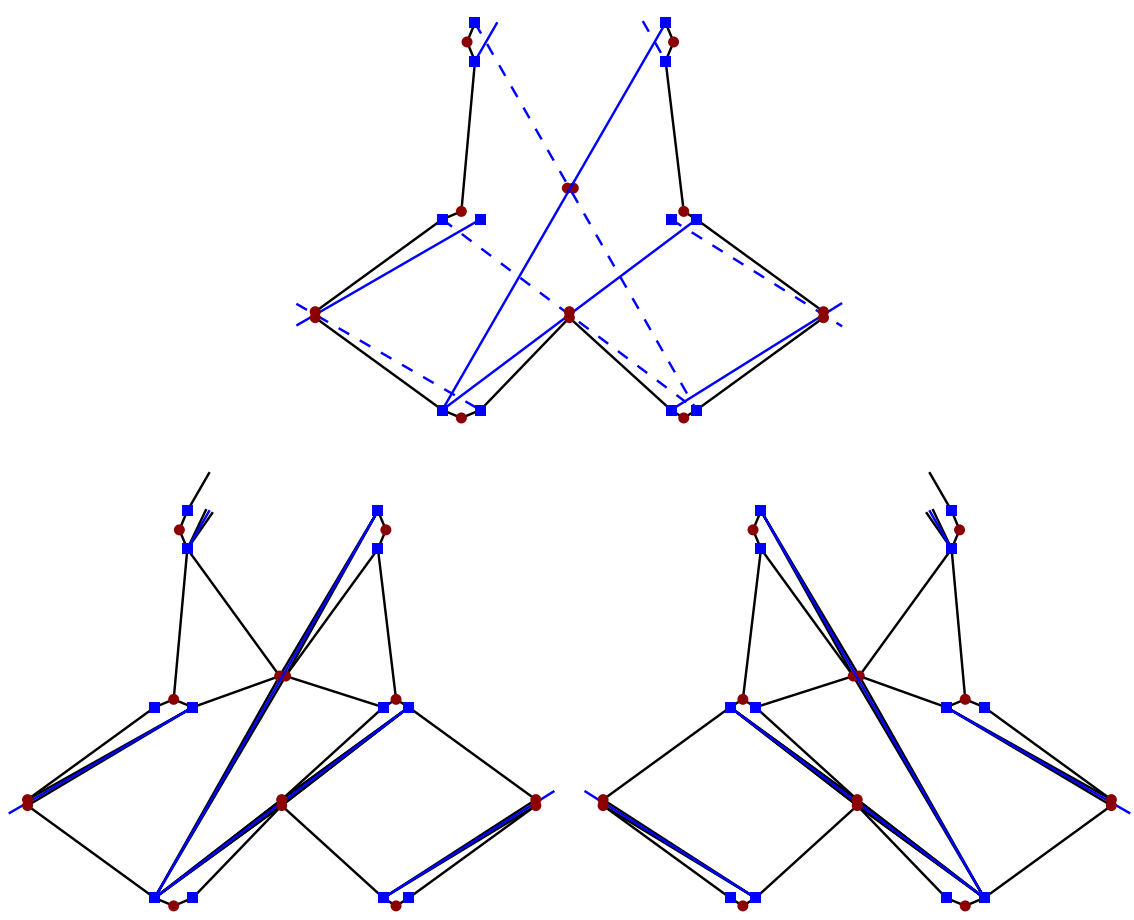

Fig. 3. A variable gadget (top), showing the possible set of blue diagonals (solid and dashed). It "splits" an edge, propagating the truth value it carries. Again, the close red points are only separated by two segments between blue points. Note that the upper edge of the incidence graph carries the negated value, which has to be compensated by adding a negation gadget along it Two possible quadrangulation are shown at the bottom.

boundary. (We thus have an even number of vertices). For the reduction, we are free to choose a polynomial number of Steiner points in the interior of the polygon, whose color we then choose according to the quadrangulation. There are several papers on quadrangulations using Steiner points, in general trying to minimize their number (which is not our concern here). We were not able to find the exact required result in the references (e.g., the convex quadrangulation in Ref. 7 uses Steiner points on the polygon boundary), but it easily follows from the following considerations. Note that any non-convex quadrilateral can be transformed into five convex ones by adding four Steiner points in the vicinity of the only diagonal. As any polygon with an even number of vertices can be quadrangulated using $O(n)$ inner Steiner points ${ }^{4}$, we have this last ingredient for our reduction. After adding these Steiner points to our construction, there is a bichromatic convex quadrangulation of our point set if and only if the corresponding planar 3-SAT instance is satisfiable. Finally, let us remark that the points can be placed in general position using coordinates of polynomial size. Before placing two "arbitrarily" close red points, inspecting the 

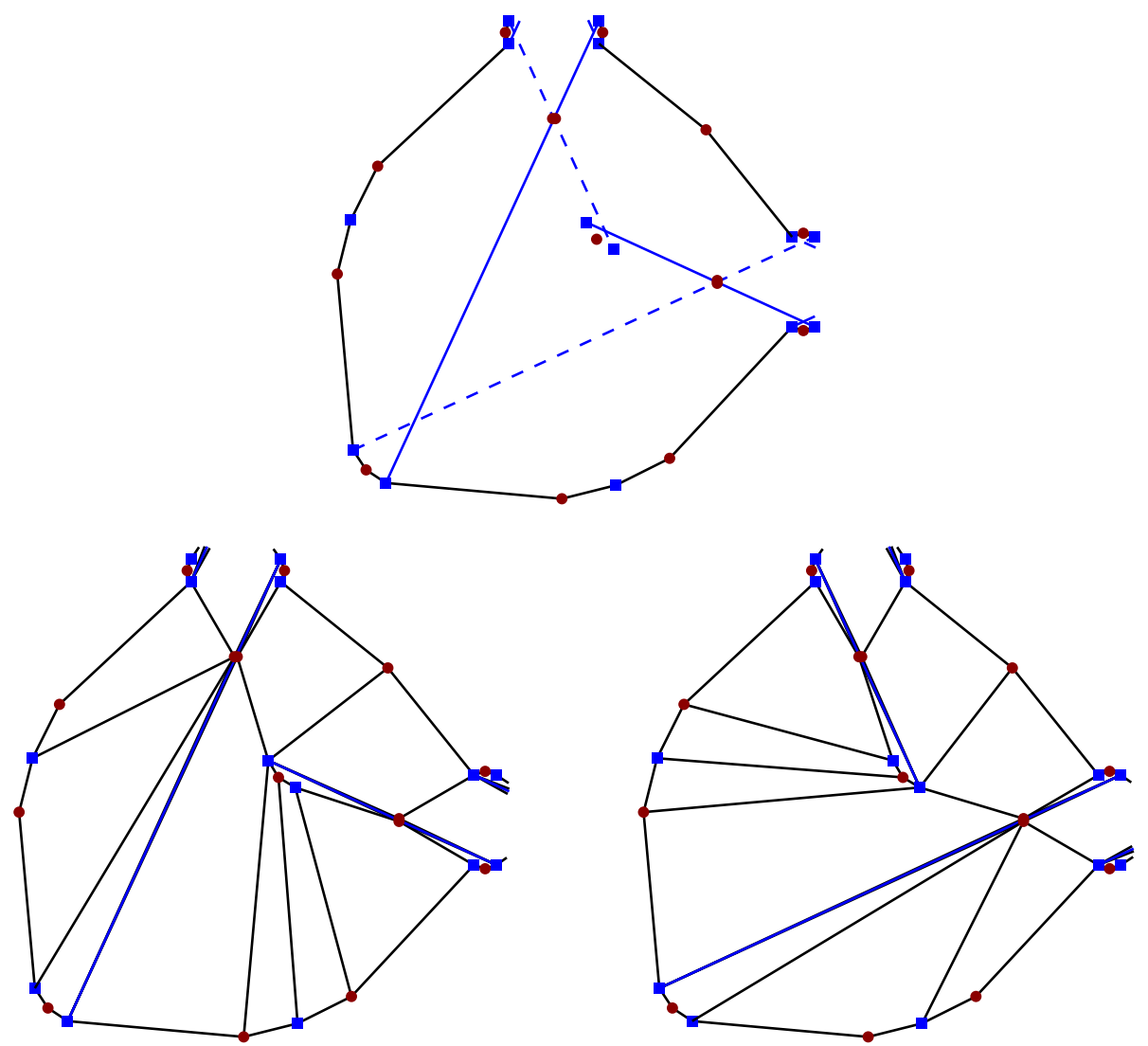

Fig. 4. A bend gadget to allow routing the edges orthogonally. Possible quadrangulations for the two truth settings are shown at the bottom.

arrangement of all segments spanned by two blue points allows us to place the points sufficiently close to each other (possibly after perturbing the set).

Theorem 2. Given a set of red and blue points, it is NP-complete to decide whether there is a valid convex quadrangulation of that point set.

\section{Conclusion}

The problem of constructing a convex quadrangulation of a point set is NP-hard when we add additional constraints. The bichromatic setting is a way to forbid certain edges in the quadrangulation. For our reduction, it is sufficient to forbid those between the close red points in the gadgets. We do not know how to achieve this in an unconstrained setting, which would allow us to apply our reduction idea in the unresolved monochromatic case. 

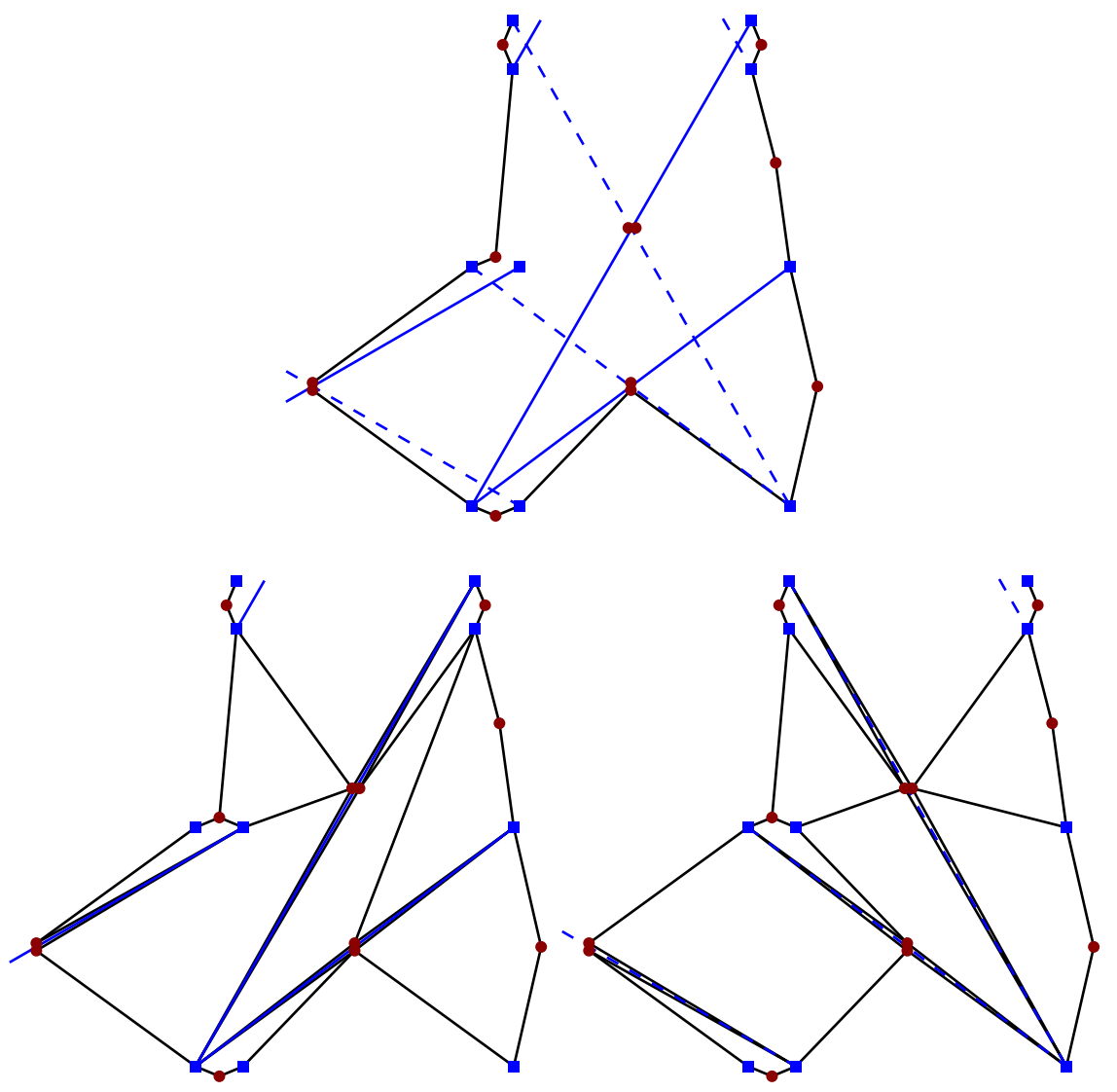

Fig. 5. A negation gadget. The structure is similar to a variable gadget. It performs a bend, which may have to be compensated by up to three bend gadgets. Two possible quadrangulations for the different truth values carried by the edge are shown.

\section{References}

1. V. Alvarez and A. Nakamoto. Colored quadrangulations with Steiner points. In J. Akiyama, M. Kano, and T. Sakai, editors, TJJCCGG 2012, volume 8296 of LNCS, pages 20-29. Springer, 2012.

2. V. Alvarez, T. Sakai, and J. Urrutia. Bichromatic quadrangulations with Steiner points. Graphs Combin., 23:85-98, 2007.

3. P. Bose and G. Toussaint. Characterizing and efficiently computing quadrangulations of planar point sets. Comput. Aided Geom. Des., 14(8):763 - 785, 1997.

4. D. Bremner, F. Hurtado, S. Ramaswami, and V. Sacristán. Small strictly convex quadrilateral meshes of point sets. Algorithmica, 38(2):317-339, 2004.

5. C. Cortés, D. Garijo, M. A. Garrido, C. I. Grima, A. Márquez, A. Moreno-González, J. Valenzuela, and M. T. Villar. Reporting bichromatic segment intersections from point sets. Int. J. Comput. Geometry Appl., 22(5):421-438, 2012. 


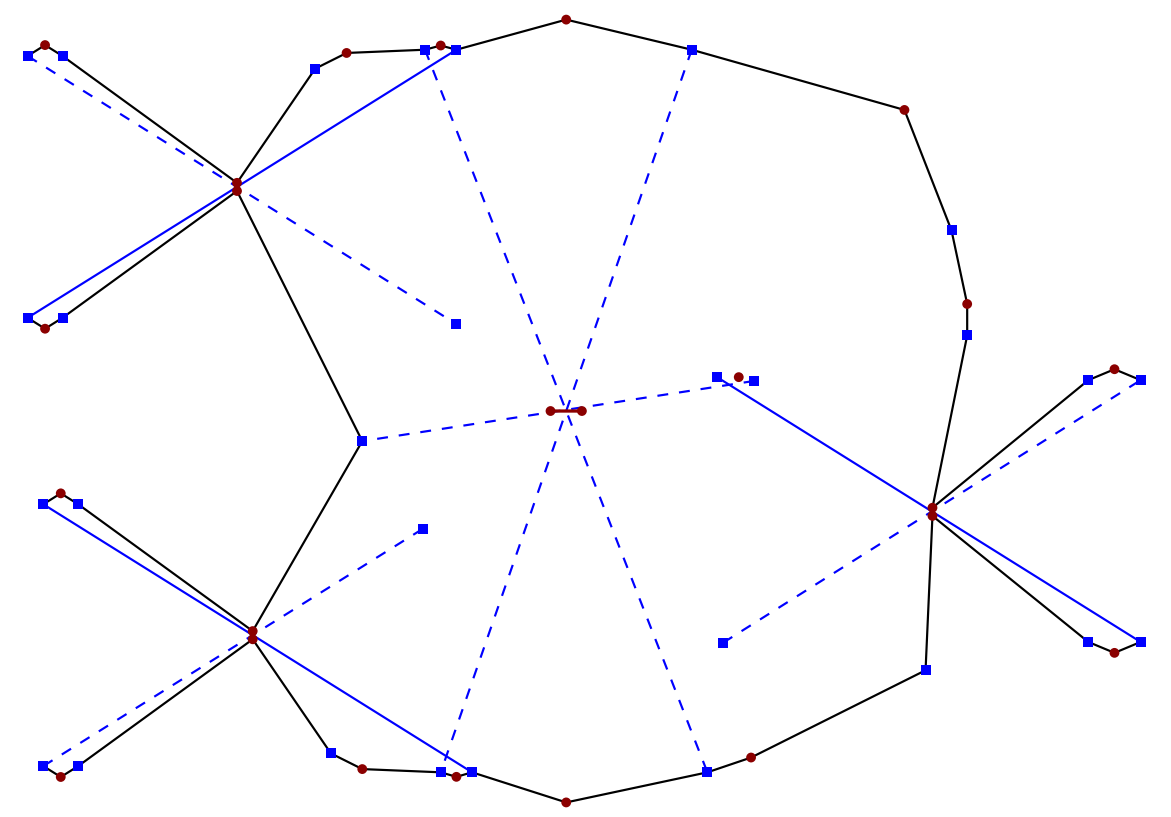

Fig. 6 . The clause gadget. The two red points in the middle connected by a red segment are closer than drawn. The only combination of blue diagonals for the link gadgets that cannot happen is the one including all three solidly drawn segments. We negate the top-left edge to make this the configuration with all literals set to false.

6. C. Cortés, A. Márquez, A. Nakamoto, and J. Valenzuela. Quadrangulations and 2colorations. In Proc. $21^{\text {st }}$ Euro CG, pages 65-68, 2005.

7. H. Everett, W. J. Lenhart, M. Overmars, T. C. Shermer, and J. Urrutia. Strictly convex quadrilateralizations of polygons. In Proc. 4th CCCG, pages 77-82, 1992.

8. T. Fevens, H. Meijer, and D. Rappaport. Minimum convex partition of a constrained point set. Discrete Appl. Math., 109(12):95 - 107, 2001.

9. V. M. Heredia and J. Urrutia. On convex quadrangulations of point sets on the plane. In CJCDGCGT, volume 4381 of $L N C S$, pages 38-46, 2005.

10. S. Kato, R. Mori, and A. Nakamoto. Quadrangulations on 3-colored point sets with steiner points and their winding numbers. Graphs Combin., 30(5):1193-1205, 2014.

11. D. E. Knuth and A. Raghunathan. The problem of compatible representatives. SIAM J. Discret. Math., 5(3):422-427, 1992.

12. D. Lichtenstein. Planar formulae and their uses. SIAM J. Comput., 11(2):329-343, 1982.

13. A. Lubiw. Decomposing polygonal regions into convex quadrilaterals. In Proc. $1^{\text {st }}$ SoCG, pages 97-106, 1985.

14. S. Ramaswami, P. Ramos, and G. Toussaint. Converting triangulations to quadrangulations. Comput. Geom., 9(4):257 - 276, 1998.

15. T. Schiffer, F. Aurenhammer, and M. Demuth. Computing convex quadrangulations. Discrete Appl. Math., 160(45):648 - 656, 2012.

16. G. T. Toussaint. Quadrangulations of planar sets. In WADS, volume 955 of $L N C S$, pages 218-227, 1995. 


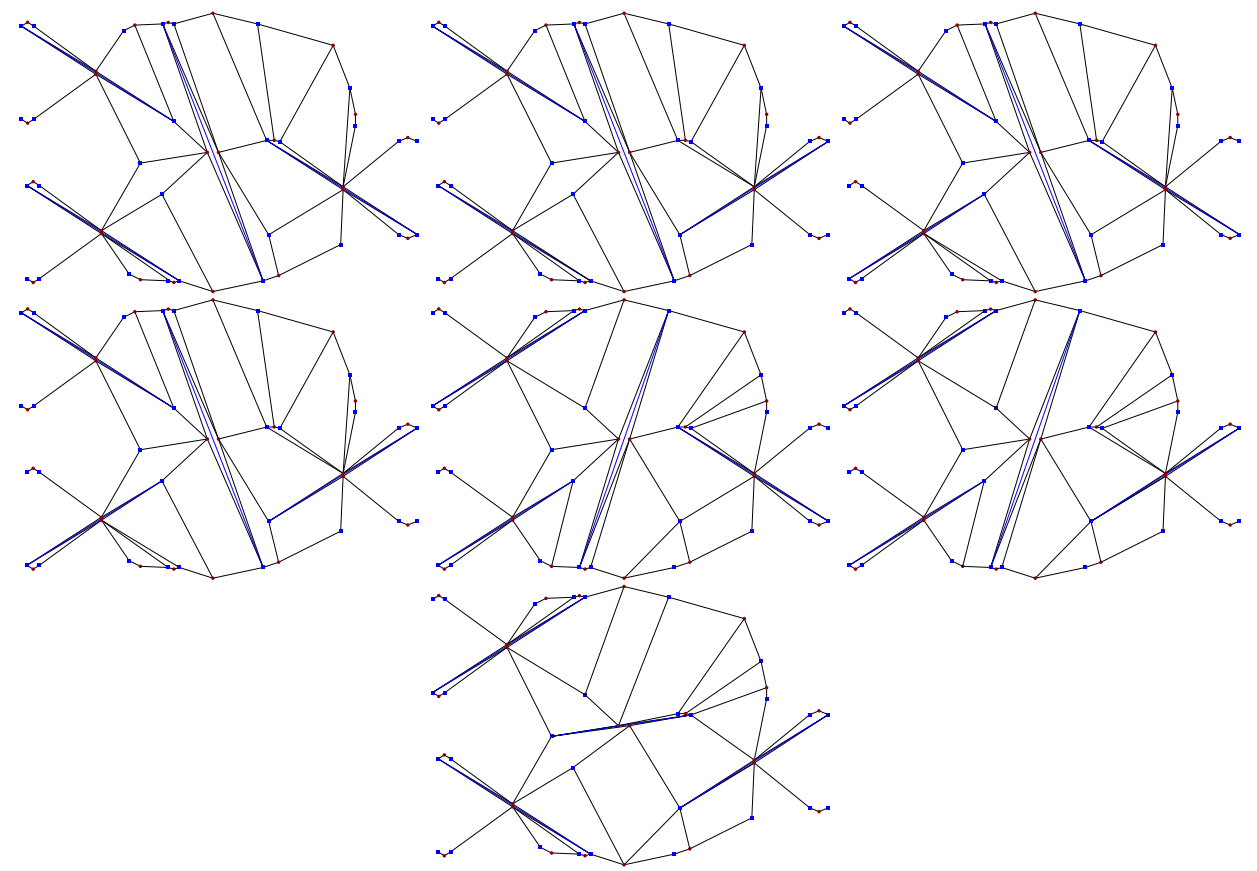

Fig. 7. Quadrangulations for each valid input to the clause gadget. 